\title{
Multifrequency Acoustic Levitation
}

\section{Puranen, Tuomas}

IEEE

2019

Puranen , T , Helander , P , Meriläinen , A, Maconi , G , Penttilä , A , Gritsevich , M , Kassamakov , I, Salmi , A, Muinonen , K \& Haeggström , E 2019 , Multifrequency Acoustic Levitation . in 2019 IEEE International Ultrasonics Symposium (IUS) . IEEE International Ultrasonics Symposium , IEEE , pp. 916-919, IEEE International Ultrasonics Symposium , Glasgow , United Kingdom , 06/10/2019 . https://doi.org/10.1109/ULTSYM.2019.8926200

http://hdl.handle.net/10138/322170

https://doi.org/10.1109/ULTSYM.2019.8926200

unspecified

acceptedVersion

Downloaded from Helda, University of Helsinki institutional repository.

This is an electronic reprint of the original article.

This reprint may differ from the original in pagination and typographic detail.

Please cite the original version. 


\title{
Multifrequency Acoustic Levitation
}

\author{
Tuomas Puranen ${ }^{1}$, Petteri Helander ${ }^{1}$, Antti Meriläinen ${ }^{1}$, Göran Maconi ${ }^{1}$, Antti Penttilä ${ }^{1}$, \\ Maria Gritsevich $^{1,2,3}$, Ivan Kassamakov ${ }^{1}$, Ari Salmi ${ }^{1}$, Karri Muinonen ${ }^{1,3}$, Edward Hæggström ${ }^{1}$ \\ ${ }^{1}$ Department of Physics, University of Helsinki, Helsinki, Finland \\ ${ }^{2}$ Institute of Physics and Technology, Ural Federal University, Ekaterinburg, Russia \\ ${ }^{3}$ Finnish Geospatial Research Institute FGI, Masala, Finland \\ Email: tuomas.puranen@ helsinki.fi, ari.salmi@helsinki.fi
}

\begin{abstract}
Acoustic levitation permits non-contact particle manipulation. The position and orientation of the levitated particle can be controlled by altering the acoustic field. Existing acoustic levitators have employed a single frequency which limits the types of acoustic traps that can be created. The use of multiple frequencies makes it possible to control the forces acting on a particle independently in all directions. We predict theoretically the forces acting on particles placed in the acoustic fields created with multiple coexisting frequencies. To realize the fields, we constructed a 450-channel phased array acoustic levitator with individual frequency, phase, and amplitude control for each channel.
\end{abstract}

Index Terms-acoustic levitation, acoustic radiation force, ultrasonics, phased arrays

\section{INTRODUCTION}

Acoustic levitation can be used for non-contact sample handling in for example chemistry and life sciences [1]. The method is suitable for millimeter scale liquid or solid samples. State-of-the-art acoustic levitators have been realized using large phased arrays operating in ultrasonic frequencies. Typically, the phased array levitators use a single static acoustic field that is changed slowly over time if needed [2]. This limits the kinds of traps, i.e. points of stable levitation, that can be created. There has been attempts to counter this, for example, in [3] they have used time multiplexing of two different fields to lock sample orientation.

Here we demonstrate acoustic levitation with two simultaneous frequencies. This gives us the advantages of time multiplexing different fields without any ill-defined transitions between them. Our phased array levitator was used to demonstrate two multifrequency fields: a two-way vortex and a multidirectional trap. In addition, we characterized the levitation fields and built a simulation model to predict the acoustic forces in the traps.

\section{Methods}

\section{A. Theoretical background}

The dynamics of the levitated particle are determined by gravity and acoustic radiation force. An estimate for the acoustic force can be obtained using Gor'kov potential [4]:

$$
U_{\text {Gor'kov }}=V_{p}\left(\frac{1}{2 \rho c^{2}}\left\langle P^{2}\right\rangle-\frac{3}{4} \rho\left\langle V^{2}\right\rangle\right),
$$

where $V_{p}$ is the particle volume, $\rho$ is the density of air, and $c$ is the speed of sound in air. The value depends on the square of the RMS pressure $\left\langle P^{2}\right\rangle$ and on the square of the RMS air particle velocity $\left\langle V^{2}\right\rangle$. Time averaging is done in the time scale where particle dynamics happen, on the order of $10 \mathrm{~ms}$ (gravitational acceleration on the length scale of the acoustic wavelength), whereas the period of the acoustic wave is on the order of $10 \mu \mathrm{s}$. This makes it possible to use pressure fields that change in time while keeping the RMS value constant. An examples of this are time multiplexed fields where one rapidly switches between different aoucstic fields. An alternative method is to use multiple simultaneous frequencies. The frequencies must be chosen so that the beat frequency is high enough for the inertia to prevent erratic movement. The squared RMS value of a field with two frequencies $\left(\omega_{1}, \omega_{2}\right)$ and pressure amplitudes $\left(P_{1}, P_{2}\right)$ is

$$
\left\langle\left(P_{1} \cos \left(\omega_{1} t\right)+P_{2} \cos \left(\omega_{2} t\right)\right)^{2}\right\rangle=\frac{1}{2}\left(P_{1}^{2}+P_{2}^{2}\right) .
$$

Here the interference term averages out and it is possible to handle the fields separately. This is also true for more elaborate models of the acoustic radiation force.

The single frequency fields offer limited acoustic control as there exists a direction where the directional derivative of pressure is zero and therefore no force is exerted in this direc-

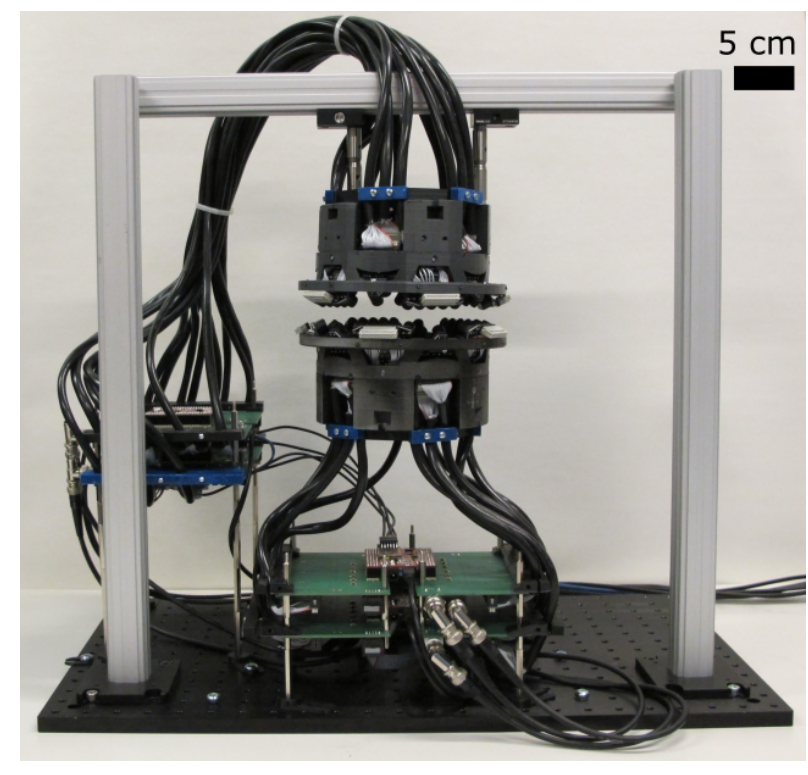

Fig. 1. Phased Array Levitator 
tion. The multifrequency acoustic traps have advantages over single frequency fields as they allow projecting simultaneous and independent forces on the levitated object.

\section{B. Phased Array Levitator}

The phased array levitator consists of 450 transducers (Multicomp MCUST10P40B07RO, $\varnothing=10 \mathrm{~mm}, 40 \mathrm{kHz}$ nominal frequency) positioned in two hemispheres (Fig. 1). The PC computes the required phase and amplitude for each transducer and sends the information to four FPGAs by the UART protocol. The FPGAs generate a square wave signal for each channel. These are amplified by level-shifter ICs to a maximum of $30 \mathrm{Vpp}$. The output pressure amplitude is controlled by the duty cycle of the square wave and the narrowband nature of the transducers makes the output sinusoidal. The phase and frequency can bet set independently for each transducer with a resolution of $\pi / 50$ and $10 \mathrm{~Hz}$, respectively.

\section{Acoustic Field Measurements}

To validate the operation of the phased array we measured the shape of the generated acoustic field. The measurement was performed by mounting a transducer on a three-axis translation stage. This receiving transducer was identical to the ones used in the phased array. A needle-like waveguide was attached onto the transducer to achieve $1 \mathrm{~mm}$ spatial resolution. The plane of interest was scanned and the amplitude and phase of the acoustic field were recorded at each point.

In addition, the pressure measurement was used to calibrate the levitator before use. Measuring the channel-vice acoustic pressure in the intended levitation spot allowed us to compensate for unidealities in the geometry and transducers.
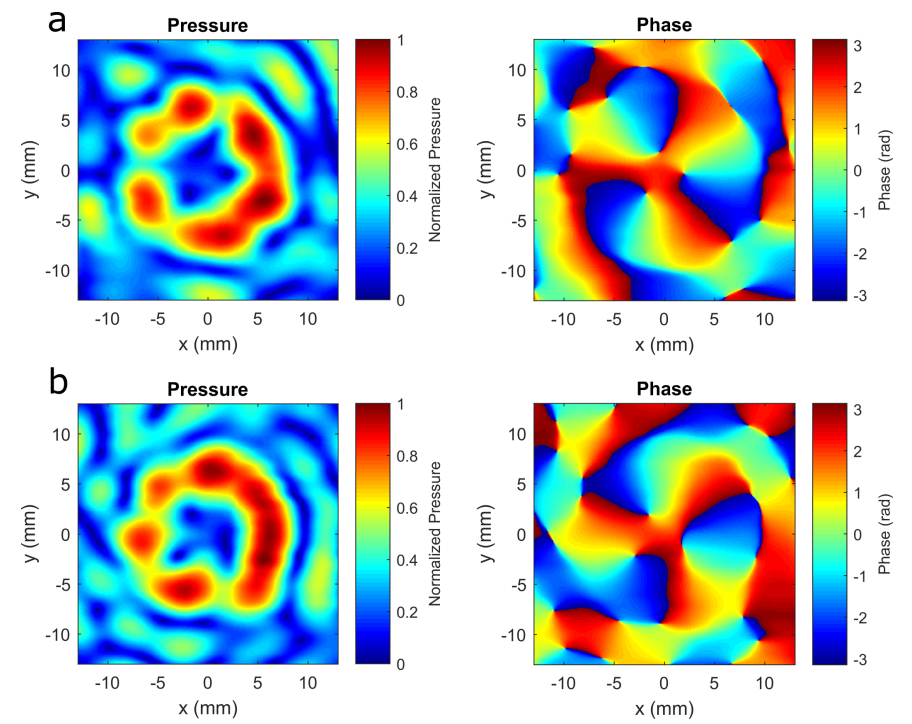

Fig. 2. Double vortex trap. The measured amplitude and phase of the two counter-rotating vortices with chirality of a) 3 and b) -3 .

\section{Multifrequency Acoustic Fields}

We generated two different traps to demonstrate the benefits of using multiple frequencies. The first is a double vortex trap that is constructed from two counter-rotating acoustic vortices with different frequencies. Acoustic vortices can trap particles that are larger than the wavelength of the sound, but they exert a torque on the particle which makes the levitation unstable [5]. By applying equally powerful but opposite vortices the torque cancels out and stable levitation can be achieved. This acoustic field was created using the lower hemisphere of levitator. The transducers were divided into two groups, every other around the polar angle $\phi$, one for each frequency $\left(f_{1}=39.6 \mathrm{kHz}\right.$ and $\left.f_{2}=40.4 \mathrm{kHz}\right)$. The relative complex amplitudes for the transducers were set as

$$
\begin{aligned}
& p_{n}^{f_{1}}=e^{i C_{1} \phi_{n}} \text { and } \\
& p_{n}^{f_{2}}=e^{i C_{2} \phi_{n}},
\end{aligned}
$$

where $\phi_{n}$ is the polar angle of the nth transducer, and $C_{1}=$ 3 and $C_{2}=-3$ are the chiralities of the vortices. The cross sections of generated pressure field are presented in Fig. 2. The vortices are visible as the large pressure nodes in the amplitude map. The coaxial vortices overlap precisely.

The second trap is an upright orientation trap. In general, the asymmetries in the trap turn the particle into the most energetically favorable orientation where the longest dimension aligns with the weakest axis of the trap. To counteract gravity the trap needs to be strong in the z-direction. The upright orientation in the single frequency configuration would require the directional derivative of pressure to be zero along the z-direction. This limits the achievable particle orientations to ones where the longest dimension is not in the direction of gravity. We circumvent this limitation by making an asymmetric vortex
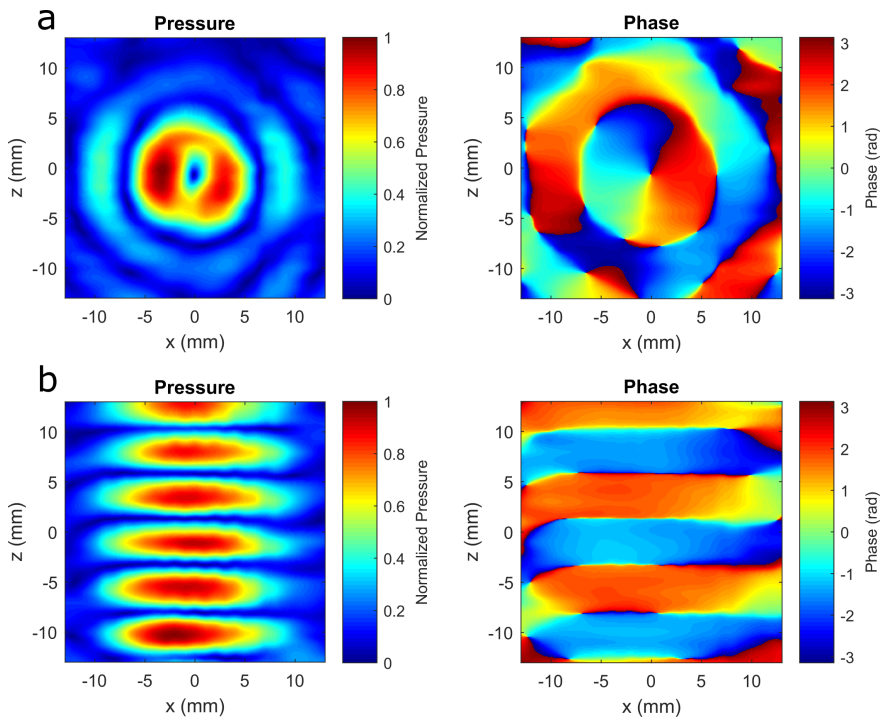

Fig. 3. Upright orientation trap. a) the measured amplitude and phase of an asymmetric acoustic vortex with chirality of 1. b) the measured standing acoustic field in $\mathrm{z}$-direction. 

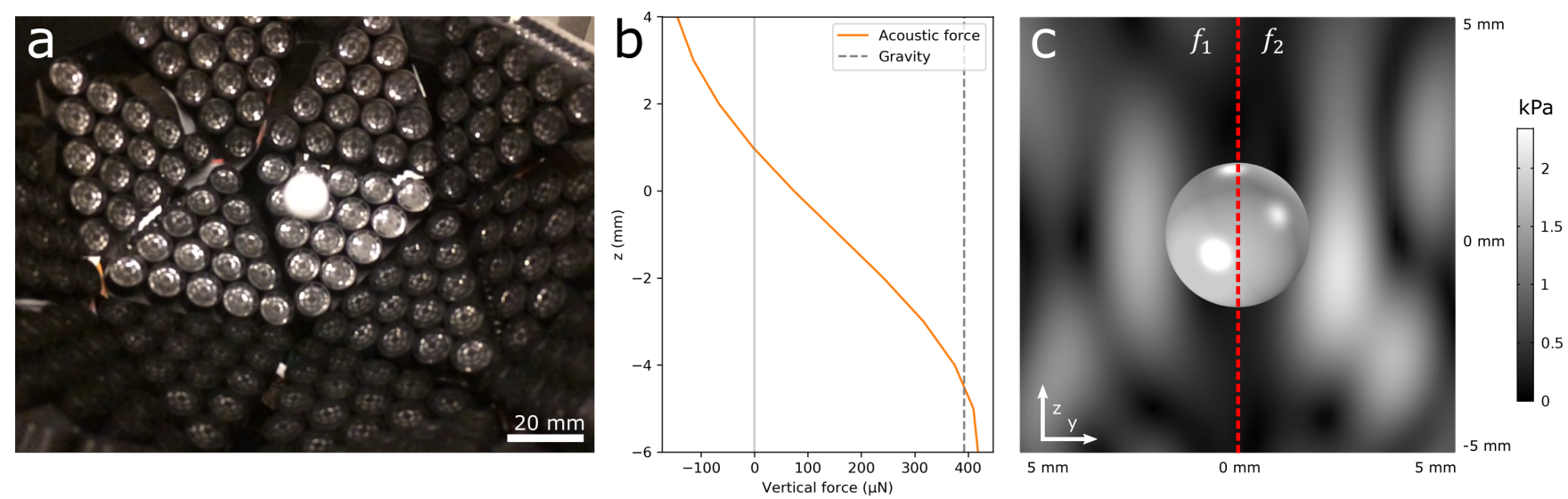

Fig. 4. Double vortex trap. a) Image of levitator in operation. b) Simulated force along the z-direction. c) Side view of the RMS pressure for both frequencies divided by the red line

that forces the orientation of the particle to be upright and by then using a second field with a different frequency to create a trap in the z-direction. The trap was created using both hemispheres, with two thirds of the transducers creating the vortex $\left(f_{1}=40.4 \mathrm{kHz}\right)$ and one third creating the standing wave $\left(f_{2}=39.6 \mathrm{kHz}\right)$. The amplitudes were set as

$$
\begin{aligned}
& p_{n}^{f_{1}}=e^{i C \phi_{i}}\left(\frac{3}{4}+\frac{1}{4} \sin ^{2} \phi_{n}\right) \text { and } \\
& p_{n}^{f_{2}}=0.3 \cdot \operatorname{sign}\left(z_{n}\right),
\end{aligned}
$$

where $z_{n}$ is the vertical position of the nth transducer and $C=1$ is the chirality. The amplitude of the standing wave is limited since otherwise its torque would exceed the one exerted by the vortex field and force the sample into horizontal orientation. The amplitude coefficients $\left(\frac{3}{4}, \frac{3}{4}\right.$ and 0.3$)$ were chosen so that this condition is fulfilled. The values were iterated to reach optimal trapping.

The measured field, Fig. 3a, shows the asymmetry of the vortex in the xy-plane. The vertical standing wave is seen in
Fig. 3B. The sample's longest dimension needs to be smaller than $3 \mathrm{~mm}$ to fit inside the pressure node.

\section{RESUlTS \& DISCUSSION}

The double vortex trap was used to levitate an expanded polystyrene (EPS) sample $(\varnothing=10 \mathrm{~mm})$, shown in Fig 4a. The vertical resonance frequency of the sample was obtained from a video and the trap stiffness was determined to be $45 \mathrm{~N} / \mathrm{mm}$. A simulation model, described in [6], was built to characterize the trap. Figs. $4 \mathrm{~b}$ and $4 \mathrm{c}$ show the cross section of the RMS pressure field for the double vortex trap and the $\mathrm{z}$ dependency of the force, respectively. The pressure amplitude was fixed using the measured stiffness value. The simulated force shows that the trap is formed at the intended location and that the levitation happens below the acoustic equilibrium position due to gravity.

Levitation in the upright orientation is demonstrated in Fig. 5a. Fig. 5b shows the simulated z-dependency of the upright orientation trap with and without the second field $\left(f_{2}\right)$. The
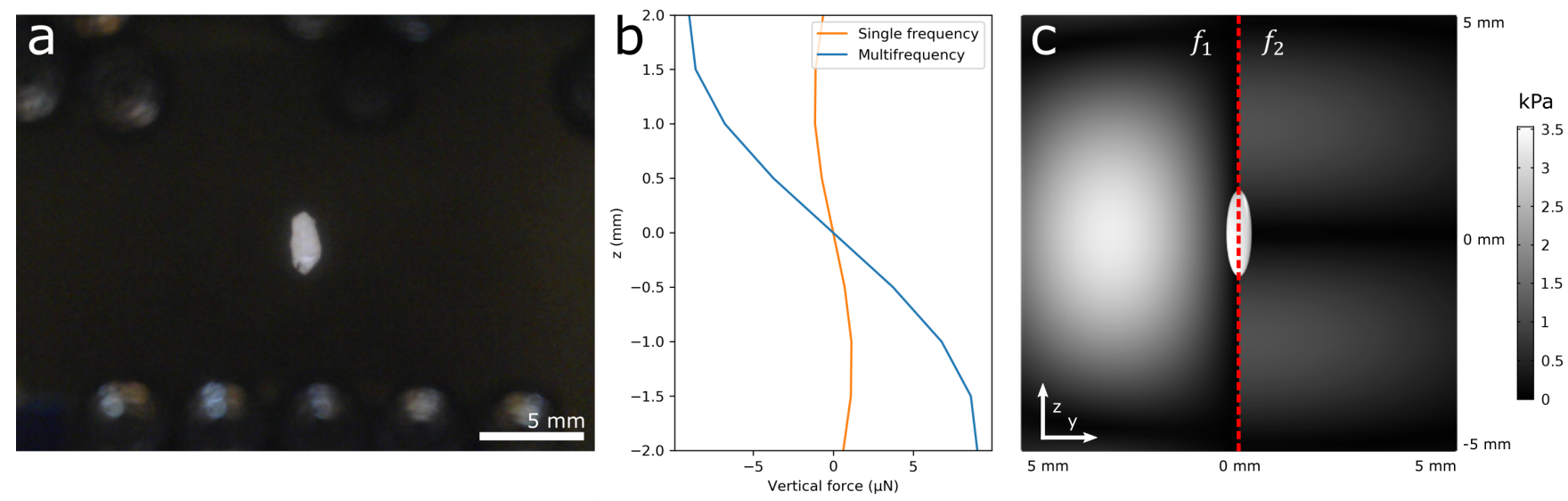

Fig. 5. Upright orientation trap. a) The levitated sample in expected orientation. b) Simulated force along the z-direction with and without the auxiliary standing wave. c) Side view of the RMS pressure for both frequencies divided by the red line. 
two-frequency trap increases the stiffness considerably and enables levitation of heavier objects.

\section{COnclusions}

We have shown acoustic traps that cannot be created using single frequency phased arrays. These new multifrequency traps levitate samples as predicted by theory and simulations. The use of multiple frequencies extends the capabilities of acoustic levitation into new types of traps.

\section{REFERENCES}

[1] S. Santesson and S. Nilsson, "Airborne chemistry: acoustic levitation in chemical analysis," Analytical and bioanalytical chemistry, vol. 378, no. 7, pp. 1704-1709, 2004.

[2] A. Marzo, S. A. Seah, B. W. Drinkwater, D. R. Sahoo, B. Long, and S. Subramanian, "Holographic acoustic elements for manipulation of levitated objects," Nature communications, vol. 6, p. 8661, 2015.

[3] L. Cox, A. Croxford, B. Drinkwater, and A. Marzo, "Acoustic lock: Position and orientation trapping of non-spherical sub-wavelength particles in mid-air using a single-axis acoustic levitator," Applied Physics Letters, vol. 113 , no. 5, p. 054101, 2018.

[4] L. P. Gor'kov, "On the forces acting on a small particle in an acoustical field in an ideal fluid," in Sov. Phys. Dokl., vol. 6, 1962, pp. 773-775.

[5] A. Marzo, M. Caleap, and B. W. Drinkwater, "Acoustic virtual vortices with tunable orbital angular momentum for trapping of mie particles," Physical review letters, vol. 120, no. 4, p. 044301, 2018.

[6] P. Helander, T. Puranen, G. Maconi, A. Penttilä, M. Gritsevich, I. Kassamakov, A. Salmi, K. Muinonen, and E. Hæggström, "Simulating acoustic orientation trapping for stable levitation," unpublished. 